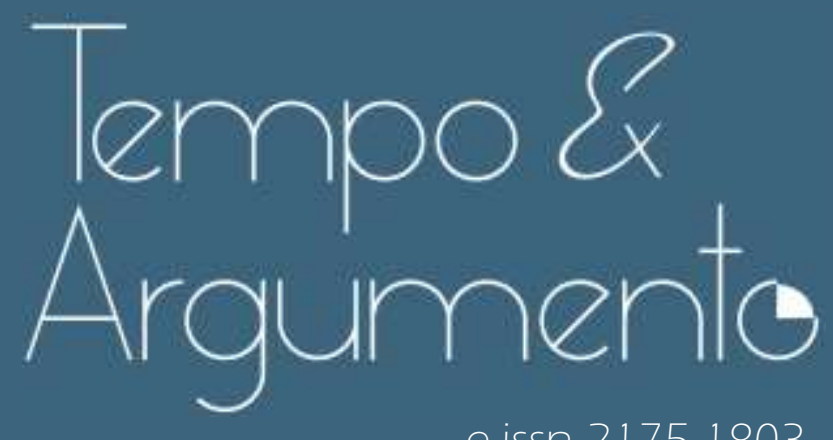

e-issn 2175-1803

\title{
Recurso de la acción de tutela por derecho a la salud mental en la jurisprudencia colombiana, 2000-2019
}

- Jairo Gutiérrez Avendaño

Doctor en Ciencias Sociales y Humanas por la Universidad Nacional de Colombia sede Medellín. Docente integrante del grupo Estudios de Fenómenos Psicosociales, Universidad Católica Luis Amigó. Integrante de la Red Colombiana de Historia de la Salud Mental.

Medellín - COLOMBIA

scienti.minciencias.gov.co/cvlac

jairo.gutierrezav@amigo.edu.co

(1D) orcid.org/0000-0002-0642-0722

Edwin Herazo

Canditado a Doctor en Salud Pública por la Universidad Nacional de Colombia sede Bogota. Director del Instituto de Investigación del Comportamiento Humano.

Integrante de la Red Colombiana de Historia de la Salud Mental.

Bogotá - COLOMBIA

scienti.minciencias.gov.co/cvlac

eh@comportamientohumano.org

(1D) orcid.org/0000-0002-9461-7997

Para citar este articulo (ABNT):

GUTIÉRREZ AVENDAÑO, Jairo; HERAZO, Edwin. Recurso de la acción de tutela por derecho a la salud mental en la jurisprudencia colombiana, 2000-2019.

Tempo e Argumento, Florianópolis, v. 13, n. 33, e0 1 17, maio/ago. 2021.

do: http://dx.doi.org/10.5965/2175180313332021e0117

Recebido: $27 / 11 / 2020$

Aprovado: 29/04/2021 


\title{
Recurso de la acción de tutela por derecho a la salud mental en la jurisprudencia colombiana, 2000-2019
}

\begin{abstract}
Resumen
Objetivo: analizar la eficacia de la acción de tutela por derecho a la salud mental en la jurisprudencia colombiana, 2000-2019. Metodología: estudio cualitativo de enfoque hermenéutico, mediante análisis descriptivo - retrospectivo de 85 sentencias de tutela revisadas y falladas en segunda instancia por la Corte Constitucional de Colombia. Conclusiones: se evidencia que la tendencia ha sido la de conceder el amparo solicitado y que los demandantes hacen parte de la población con más vulnerabilidad. Se destaca la reiteración de jurisprudencia por un mismo problema jurídico; en particular, el acceso a la prestación de servicios excluidos del Plan obligatorio de Salud (POS). Este mecanismo tutelar, además de ayudar a garantizar la atención de los usuarios, es un indicador de la eficacia y eficiencia del servicio, que ha sentado precedentes en la jurisprudencia para revisar, confirmar y derogar actuaciones administrativas y técnicas que limitan o vulneran los derechos a la salud, la vida digna y la seguridad social.
\end{abstract}

Palabras clave: Acción de tutela; Salud mental; Vida digna; Seguridad social; Discapacidad mental.

\section{Appeal of the protection action for the right to mental health in Colombian jurisprudence, 2000-2019}

\begin{abstract}
Objective: to analyze the efficacy of the tutela action for the right to mental health in Colombian jurisprudence, 2000-2019. Methodology: qualitative study of hermeneutic approach, through descriptive - retrospective analysis of 85 judgments reviewed and ruled in second instance by the Constitutional Court of Colombia. Conclusions: it is evident that the tendency has been to grant the requested protection and that the plaintiffs are part of the population with more vulnerability. Is remarkable the reiteration of jurisprudence for the same legal problem; in particular, access to the provision of services excluded from the Mandatory Health Plan (POS). This action for protection, in addition to helping to guarantee the attention of users, is an indicator of the efficacy and efficiency of the service, which has set precedents in jurisprudence to review, confirm and repeal administrative and technical actions that limit or violate the rights to health, a dignified life and social security.
\end{abstract}

Keywords: Action for protection; Mental health; Dignified life; Social security; Mental disability. 


\section{Introducción}

La República de Colombia introdujo en la vigente Constitución Política (CP) de 1991 la acción de tutela, que es un mecanismo de defensa judicial, preferente y sumario, al que puede acudir cualquier persona cuando sus derechos fundamentales resulten vulnerados o amenazados por la acción u omisión en que incurra cualquier autoridad pública o un particular y no exista otro mecanismo de defensa judicial que permita su protección idónea y efectiva. Por regla general, la tutela no procede contra providencias judiciales en virtud de los principios de autonomía judicial y seguridad jurídica (REPÚBLICA DE COLOMBIA, 1991a; REPÚBLICA DE COLOMBIA, 1991b).

La acción de tutela goza de informalidad, en el evento que se pueda acudir personalmente a un Juzgado, allí se puede radicar directamente por escrito o verbalmente siempre y cuando sea un caso de urgencia o cuando el solicitante no sabe escribir o si es menor de edad. La solicitud debe contener: a) los nombres y apellidos completos del solicitante; b) la acción o la omisión que la motiva; c) el derecho que se considera violado o amenazado; d) el nombre de la autoridad pública, si fuere posible, o del órgano autor de la amenaza o del agravio; e) la descripción de las demás circunstancias relevantes para decidir la solicitud; f) lugar, dirección de residencia, teléfono y correo electrónico del solicitante; g) no será indispensable citar la norma constitucional infringida, siempre que se determine claramente el derecho violado o amenazado; h) anexar a la solicitud la copia de los documentos relacionados con el caso para facilitar la actividad judicial.

Si bien, la salud se consideró inicialmente en la CP como un servicio público y no expresamente un derecho fundamental, la Corte Constitucional (CC) de forma temprana se pronunció en el sentido de que la salud era un derecho que guardaba conexidad con el derecho fundamental a la vida, y por lo tanto el acceso a los servicios de salud mental podía ser considerado también un derecho y ser objeto de amparo a través de la acción de tutela, conforme a la Sentencia SU 111 de 1997 de la CC, hasta llegar a la sentencia T-760 de 2008 de la CC, la cual marcó un hito en relación con el derecho a la salud en Colombia.

Adicionalmente, al configurarse el Sistema General de Seguridad Social en Salud a partir de la Ley 100 de 1993 y la posterior definición del Plan Obligatorio 
de Salud (POS)1, mediante el Acuerdo 008 (MINISTERIO DE SALUD, 1994), se evidenció una discriminación institucional hacia la salud mental, debido a que limitaba los servicios en este campo en particular. Por ejemplo, se excluía la psicoterapia individual y la psicoterapia prolongada, cubriendo sólo la fase inicial y aguda de una enfermedad, entendida como los primeros 30 días calendario. De la misma manera, la internación por problemas o trastornos mentales no podía exceder este mismo término, en una desatención flagrante a preceptos que asumen la garantía y ejercicio de los derechos como un aspecto inherente a la salud mental (AITKEN, 2011).

Este tipo de restricciones y exclusiones, impulsaban a los ciudadanos a acudir a la acción de tutela como herramienta constitucional que hiciera valer su derecho de acceder al servicio público de salud, situación que se fue complicando y complejizando en la medida que el modelo de aseguramiento y de rentabilidad financiera se anidaba en el sistema de salud colombiano y terminaría por cooptarlo (CORCHO, 2018).

Una situación que resulta llamativa es que en todo este proceso de reorganización del sistema de salud, la primera Política Nacional de Salud Mental, promulgada en 1998, no resultó una herramienta de gobierno que contribuyera de manera fehaciente a la solución de los problemas de salud mental. Con el paso del tiempo se hicieron modificaciones al POS, mediante la Comisión de Regulación de Salud², en su Acuerdo 028 de 2011 y más recientemente al Plan de Beneficios en Salud, en su Acuerdo 029 de 2011 y en la Resolución 5857 de 2018 de MINSALUD, así como la promulgación de la Ley Estatutaria de Salud 1751 de $2015^{3}$ que consagra expresamente la salud como un derecho fundamental.

\footnotetext{
El POS actualmente se denomina Plan de Beneficios en Salud, con fundamento en la Ley Estatutaria de Salud, No. 1751 de 2015 y la Resolución 5592 de 2015 del Ministerio de Salud y Protección Social.

2 Ver: COMISIÓN DE REGULACIÓN DE SALUD. Acuerdo 029 de 2011. Metodología para la actualización integral del Plan obligatorio de salud del Sistema general de Seguridad social en Salud. Bogotá: Minsalud, 2011. Disponible en: https://www.minsalud.gov.co. Acceso en: 10 dic. 2019, e;

COMISIÓN DE REGULACIÓN DE SALUD. Acuerdo 028 de 2011. Por el cual se define, aclara y actualiza integralmente el Plan Obligatorio de Salud. Bogotá: Minsalud, 2011. Disponible en: https://www.minsalud.gov.co. Acceso en: 10 dic. 2019.

${ }^{3}$ Ver: CONGRESO DE LA REPÚBLICA. Ley Estatutaria 1751 de 2015. Por medio de la cual se regula el derecho fundamental a la salud y se dictan otras disposiciones. Diario Oficial, Bogotá, n. 49.427, 16 feb. 2015. Disponible en: https://www.minsalud.gov.co. Acceso en: 10 dic. 2019.
} 
El Estado colombiano tiene una completa normativa actualizada que ha refrendado el derecho a la salud mental, como es la Ley 1616 de $2013^{4}$ y adoptada por Resolución 4886 de 20185; sin embargo, los datos referentes a las acciones de tutela para amparar la protección de este derecho fundamental, nos muestran que la vulneración de este derecho es un problema vigente y creciente. Por las razones expuestas, el objetivo del presente artículo es analizar la eficacia de la acción de tutela por derecho a la salud mental en la jurisprudencia colombiana, en el periodo comprendido entre 2000 y 2019.

\section{Metodología}

Investigación documental de enfoque hermenéutico, mediante análisis descriptivo - retrospectivo de 85 sentencias de tutela revisadas y falladas en segunda instancia por la Corte Constitucional de Colombia, entre 2000 y 2019. Mediante la base de datos en línea de la Corte, se descargaron para el análisis las que invocaron el derecho a la salud mental, que estuvieron relacionadas con atención psiquiátrica y psicológica, situación de discapacidad mental, trastornos asociados a la adicción de SPA y salud mental en el trabajo.

Fueron excluidas las que se refirieron a efectos de relaciones exógenas y de orden físico que podrían tener un impacto psíquico o emocional, pero que no implicaron atención en salud mental propiamente dicha.

\section{Resultados}

Al final del artículo, la Tabla 5 presenta una síntesis del análisis de contenido consignado en las tutelas: por solicitud realizada, derechos invocados, problemas por resolver, diagnósticos de salud mental y los números de sentencia a que corresponden. De este modo, en las 85 tutelas falladas por la Corte

\footnotetext{
${ }^{4}$ Ver: CONGRESO DE LA REPÚBLICA. Ley 1616 de 2013 Por medio de la cual se expide la Ley de Salud Menta. Diario Oficial, Bogotá, n. 48.680, 21 enero 2013. Disponible en:

https://www.minsalud.gov.co/sites/rid/Lists/BibliotecaDigital/RIDE/DE/DIJ/ley-1616-del-21-deenero-2013.pdf. Acceso en: 10 dic. 2019.

5 Ver: MINISTERIO DE SALUD Y PROTECCIÓN SOCIAL. Resolución 4886 de 2018. Por la cual se adopta la Política Nacional de Salud Mental. Diario Oficial, Bogotá, n. 50770, 7 noviembre 2018. Disponible en:

https://www.minsalud.gov.co/Normatividad_Nuevo/Resoluci\%C3\%B3n\%20No.\%204886\%20de\% 202018.pdf. Acceso en: 10 dic. 2019.
} 
Constitucional en las que se invocó el derecho a la salud mental, se destaca que cerca del 85\% presentó reiteración de jurisprudencia, razón que influyó en la selección y revisión por parte de esta entidad judicial, conforme a lo dispuesto en los artículos 86 y 241 de la Constitución Política (REPÚBLICA DE COLOMBIA, 1991a) y 33 del Decreto 2591 (REPÚBLICA DE COLOMBIA, 1991b).

Predominó la demanda del Derecho a la salud, a la vida digna y la seguridad social (88,24\%), seguido del Derecho a la capacidad jurídica de persona en condición de discapacidad mental, así como Derechos reproductivos en persona con esta condición.

En su mayoría, revocaron y confirmaron sentencias de segunda instancia, para conceder la tutela de los derechos demandados por los accionantes (85,88\%), seguido de las que denegaron el amparo por improcedentes, principalmente debido a la carencia actual de objeto en el asunto evaluado, por hecho superado y daño consumado, así como por falta de pruebas $(14,12 \%)$. Sin embargo, se destaca que, aunque improcedentes, la Corte ordenó que se garantizara de forma oportuna y eficiente la prestación del servicio.

\section{Demandantes y demandados}

Los motivos por los que parientes y terceros recurrieron a este recurso judicial, fueron principalmente por incapacidad económica, protección personal y de cercanos (74,12\%), debido a la carga del cuidado que representa el manejo de pacientes agresivos, con intento de suicidio y porque la persona a cargo no estaba en condiciones físicas, mentales y de edad para asumir esta responsabilidad.

Otro de los motivos es por el suministro de medicamentos y servicios excluidos del Plan obligatorio de salud (POS) (14,12\%) que, igualmente, eran incosteables para las familias; entre otros motivos como: protección sexual y reproductiva, restablecimiento de derechos, mínimo vital y debilidad manifiesta.

Se registraron ocho tipos de situaciones: en primer lugar, el 54,12\% se declaró como persona con discapacidad mental, también relacionado en dos casos de privados de la libertad; seguido del 18,82\% por adicción a sustancias psicoactivas, y en dos casos con la condición de habitante de calle. En cuanto a 
los considerados en situación de riesgo, el 10,59\%, por protección personal y de cercanos, intentos de suicidio, protección sexual y reproductiva y salud mental en el trabajo (Gráfico 1)

Se presentaron cinco casos de personas privadas de la libertad (7,06 \%) y en dos de ellos con situación de discapacidad. Otras situaciones son: abandono y habitante de calle; víctima del conflicto armado en condición de desplazado; y protección de menor de edad.

De los entes demandados predominaron las acciones contra Entidades prestadoras de Salud - EPS y del régimen subsidiado (57,65\%); seguido de entidades estatales, secretarías de salud, bienestar familiar, entre otras (11,76\%); Ministerio de Defensa, Ejército y Policía - FFMM (10,59\%); Instituto Nacional Penitenciario y Carcelario - INPEC (8,24\%); entre otras entidades como Institutos prestadores de salud - IPS, Administradoras de riesgos de salud - ARS, Empresas solidarias de salud - ESS, y Empresas Sociales del Estado - ESE.

Gráfico 1 - Relación entre demandante y demandado

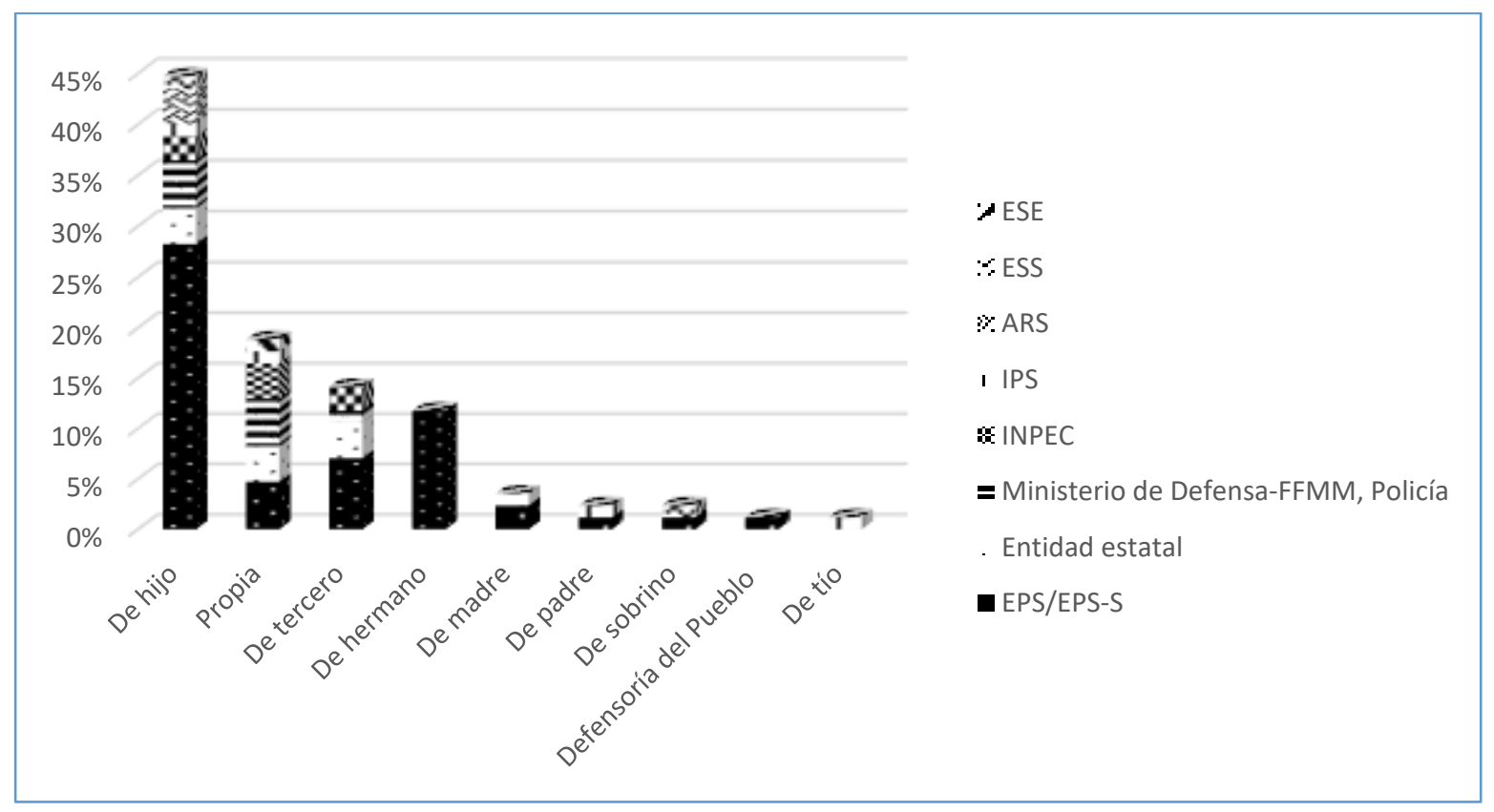

Fuente: elaboración propia, 2019.

\section{Solicitudes y problemas}

En la tabla 3 se sintetizaron 19 tipos de solicitudes en las que se identificaron 17 problemas jurídicos, de los que se destaca, en primer lugar, la 
limitación en la prestación de servicios, procedimientos y suministros excluidos del Plan obligatorio de salud (POS) (64,71\%), e incluso es el que tiene más reiteración de jurisprudencia, distribuido entre las solicitudes de internamiento en centro de rehabilitación (16,47\%) y en centro psiquiátrico (15,29\%); orden de medicamentos, elementos de aseo y nutrición (9,41\%); entre otros como, servicio especializado, exámenes; transporte especial; atención domiciliaria; hogar geriátrico; solicitud de cuidador y reintegro de costos de atención.

El segundo problema principal es el de reconocimiento de discapacidad y reintegro de atención en salud de exmilitares y expolicías (9,41\%); seguido del régimen excepcional de salud para personas privadas de la libertad provisto por el Instituto Nacional Penitenciario - INPEC (7,6\%) (Gráfico 2)

Gráfico 2 - Relación entre tipos de solicitudes y problemas jurídicos

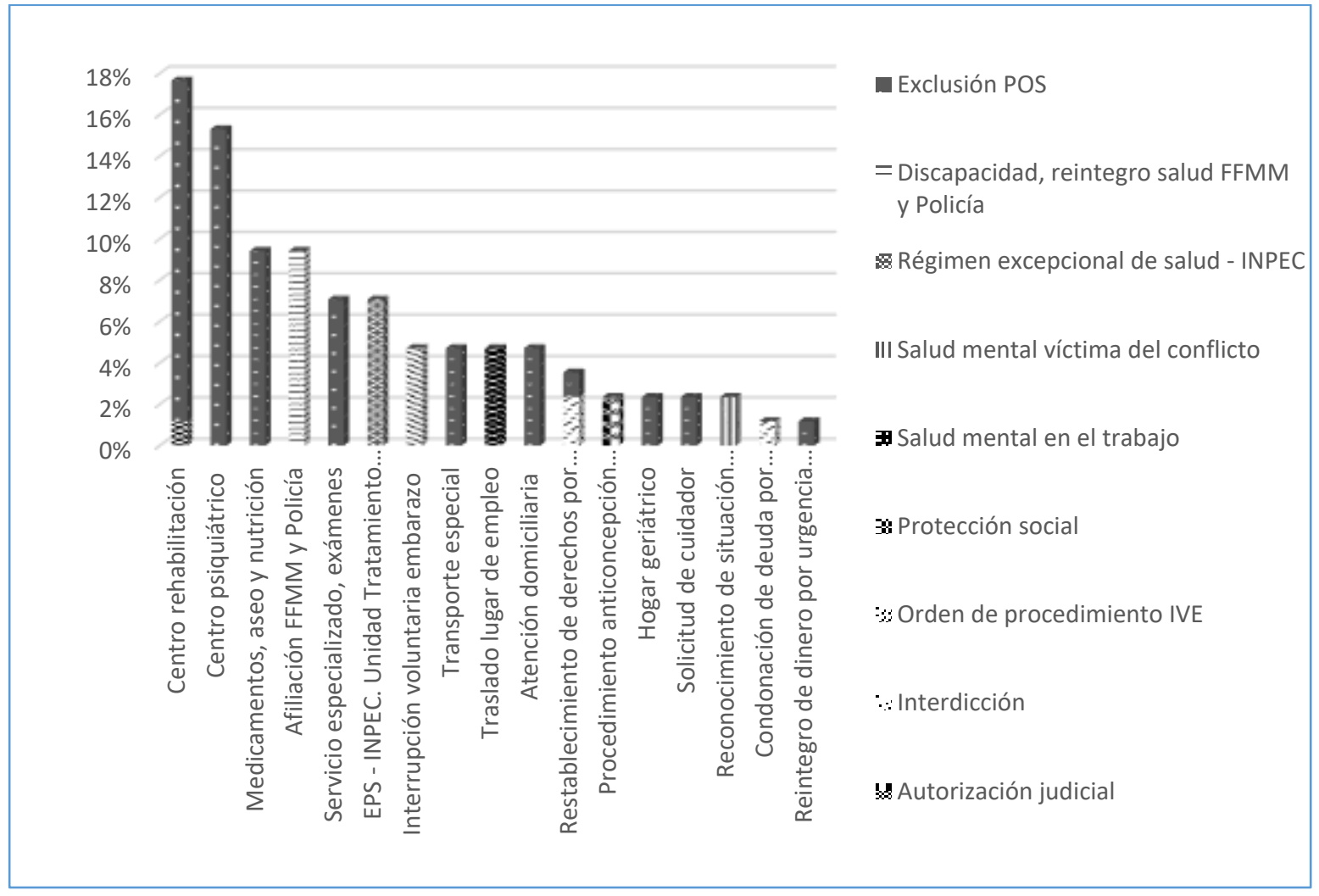

Fuente: elaboración propia, 2019.

No se precisó la relación de solicitudes y de problemas por departamentos del país, porque no es una muestra uniforme, sino una selección aleatoria que la Corte realizó; sin embargo, los cinco principales corresponden a Cundinamarca 
(25,88\%), Atlántico (10,59\%), Valle del Cauca (10,59\%), Antioquia (9,41\%) y Risaralda (7,06\%), seguidos de: Quindío, Santander, Tolima, Caldas, Bolívar, Huila, Caquetá, Córdoba, Boyacá, Casanare, Magdalena, Cauca, Meta, Norte de Santander, Amazonas y unos pocos con reserva.

Los anteriores hallazgos concuerdan con las estadísticas de peticiones, quejas, reclamos y denuncias (PQRD) de la Superintendencia Nacional de Salud, en las que se observa que la atención en Salud mental se encuentra entre los principales motivos de PQRD y tuvieron un aumento considerable entre 2018 y 2019. De hecho, se clasifica entre las enfermedades crónicas y de alto costo en Colombia.

Exceptuando las solicitudes de información a las líneas de contacto de la SuperSalud (144591), se evidenció que entre los principales cinco motivos de PQRD, se encuentra el aumento de casos que se refieren a limitaciones del POS. Asimismo, se registra un crecimiento de solicitudes por atención en las especialidades de Salud Mental. También se evidencia que, si bien disminuyó la solicitud de seguimiento, aumentó la tendencia al desacato de las tutelas por parte de entidades estatales de salud y de los prestadores del servicio (Tabla 1).

Tabla 1 - Peticiones, quejas, reclamos y denuncias PQRD

\begin{tabular}{lccc}
\hline PQRD & 2018 & 2019 & \% variación \\
\hline Total solicitudes & 353.958 & 599.375 & 69,3 \\
POS y No POS & 47.845 & 130.864 & 173,5 \\
Salud mental & 3680 & 5179 & 40,7 \\
Seguimiento tutela & 616 & 529 & $-14,1$ \\
Desacato tutela & 135 & 251 & 86 \\
Inconformidad & 2 & 11 & 4,5 \\
tutela & 2 & & \\
\hline
\end{tabular}

Fuente: Superintendencia Nacional de Salud, 2018 - 2019. Cálculo: propio

\section{Diagnósticos}

En la tabla 2, se observa que los cinco principales diagnósticos se presentaron por parejo y se encuentran dificultades para la atención en cada uno. En cuanto al retardo y discapacidad mental, ninguna de las acciones fue en representación propia, dado que esta condición produce dependencia de 
cuidadores y tutores; entre otras situaciones como la adicción a sustancias psicoactivas, persona privada de la libertad y en víctima del conflicto armado interno en condición de desplazado. En su mayoría son clientes adultos, seguido de menores de edad. En general, fueron defendidos por padres (66,77\%), hermanos $(26,67 \%)$ y un caso por tercero. La tabla 3 muestra que el retardo y la discapacidad mental también se registran entre los principales diagnósticos declarados en las PQRD interpuestas ante SuperSalud, entre 2018 y 2019.

Tabla 2 - Diagnósticos de las tutelas

\begin{tabular}{lr}
\hline Dx. & $\%$ \\
\hline Retardo y discapacidad & $17,65 \%$ \\
mental & \\
Epilepsia & $17,65 \%$ \\
Trastorno mental consumo & $15,29 \%$ \\
SPA & \\
Depresión y angustia & $15,29 \%$ \\
Esquizofrenia & $15,29 \%$ \\
Trastorno afectivo bipolar & $9,41 \%$ \\
Trastorno mental no & $3,53 \%$ \\
especificado & \\
Demencia y Alzhéimer & $2,35 \%$ \\
Psicosis & $2,35 \%$ \\
Autismo & $1,18 \%$ \\
\hline Total & $100 \%$ \\
\hline
\end{tabular}

Fuente: elaboración propia, 2019.

La epilepsia también presenta pocas solicitudes en representación propia (13,33\%), debido a que en la mayoría declararon situación de discapacidad (86,67\%). Más de la mitad son adultos (60\%), seguidos de menores de edad $(26,67 \%)$ y adultos mayores (13,33\%). Estos fueron defendidos por padres $(66,67 \%)$ y otros parientes: hermano, tío, sobrino (20\%) y un caso por tercero. La Tabla 3 muestra que la epilepsia también es uno de los principales diagnósticos declarados en las solicitudes de atención mediante PQRD interpuestas ante SuperSalud, entre 2018 y 2019.

Los trastornos por consumo de sustancias psicoactivas - SPA presentaron, de 13 solicitudes, 3 en representación propia (23,8\%). Aunque pocos se declararon en situación de discapacidad mental, la adicción en sí misma fue considerada 
una condición de protección por la alteración, deterioro mental, pérdida de calidad de vida y por ser un riesgo para sí mismos, para sus parientes y cercanos. De estos, 10 son adultos (77\%), 2 menores de edad y un adulto mayor. Fueron defendidos por padres (38,46\%), seguido de terceros, incluida la Defensoría del Pueblo (30,77\%), así como por hermanos (7,7\%).

La depresión y angustia aparecen asociadas en 13 solicitudes, de las que 8 fueron en representación propia (61,64\%), 2 en representación de hijo (5,38\%), seguido de 2 en representación de tercero y 1 de sobrino 7,69\%. De estos, 10 son adultos (77\%) y 3 menores de edad (23\%). Se consideraron 8 en situación de riesgo (61,54\%), incluyendo 4 casos por derechos reproductivos; 3 en situación de discapacidad, 1 víctima de conflicto armado interno en condición de desplazado, 1 privado de la libertad. Asimismo, la Tabla 3 muestra que la depresión y angustia se encuentran entre los principales diagnósticos declarados en las PQRD interpuestas ante SuperSalud entre 2018 y 2019.

La esquizofrenia se presentó en 13 solicitudes, de las que 7 fueron en representación de hijo (53,85\%), 2 en representación propia, 2 de hermano, 1 de padre, 1 de tercero. De estos 11 son adultos (84,62\%) y 2 adultos mayores. Se consideraron 6 en situación de discapacidad (46,15\%), 5 en adicción a SPA, 2 privados de la libertad. De igual forma, la tabla 3 muestra que la esquizofrenia es uno de los principales diagnósticos declarados en las PQRD interpuestas ante SuperSalud, entre 2018 y 2019.

Tabla 3 - Peticiones, quejas, reclamos y denuncias PQRD

\begin{tabular}{|c|c|c|c|}
\hline Dx. & 2018 & 2019 & \%variación \\
\hline Epilepsia & 2950 & 3389 & 14,9 \\
\hline Esquizofrenia & 1169 & 2029 & 73,5 \\
\hline Parkinson & 1438 & 2090 & 45,3 \\
\hline Alzheimer & 1333 & 2528 & 89,6 \\
\hline Depresión & 1137 & 780 & $-31,4$ \\
\hline Trastornos de ansiedad & 801 & 1863 & 132,6 \\
\hline Trastorno afectivo bipolar & 725 & 1615 & 122,7 \\
\hline Retraso mental & 349 & 1251 & 258,4 \\
\hline Autismo & 488 & 82 & $-83,2$ \\
\hline Demencia & 71 & 78 & 9,8 \\
\hline
\end{tabular}




\section{Discusión}

Los resultados actuales tienen continuidad y concuerdan con los aportes de Ardila (2010), quien realizó un análisis de sentencias en salud mental, emitidas por la Corte Constitucional entre 1992 y 2009, en el que concluye que las limitantes técnicas de la prestación del servicio, aunque debidas a la propias normativas, no justifican que haya limitantes valorativas o de acción, porque se evidencia que por esta vía se refuerza y perpetúa la estrechez de las mismas normas. Asimismo, se destaca el estudio de Echeverry (2013) sobre la acción de tutela como indicador de los problemas de acceso a los servicios de salud, entre 2002 y 2007, en el que sintetiza cuatro barreras narradas de forma reiterada por los usuarios, tales como:

1) Falta o insuficiencia en la oferta de algunos servicios, atribuida a demoras en la contratación, escasa disponibilidad de algunas especialidades, y supresión o disminución de servicios en el POS, por ejemplo, atención odontológica, programas de promoción y prevención, atención nutricional, sicológica y de salud mental.

2) Barreras económicas para asumir diversos costos ocasionados por servicios no incluidos en el POS, compra de medicamentos porque no son entregados en forma completa u oportuna; gastos administrativos por trámites y por fragmentación territorial de la oferta en varias IPS, ubicadas en diferentes puntos de la ciudad; búsqueda de atención particular cuando no hay resolución oportuna; y cuotas de los copagos.

3) Barreras administrativas generadas por exceso de requisitos, como son: autorizaciones de la EPS e idas y venidas entre esta y la IPS, fotocopias y constancias de negación de servicios; todo esto deviene en mayores gastos de bolsillo.

4) No resolución o resolución incompleta de la necesidad en salud, debido a la estrategia de EPS e IPS de no hacer negación explícita de servicios, difiriendo la atención en el tiempo o entregando únicamente unos servicios y otros no.

En Colombia, entre 2013 y 2018, la acción de tutela por derecho a la salud y seguridad social muestra un crecimiento en la demanda, en tanto pasó de 115.147 solicitudes en 2013, a 207.734 en 2018. Se destaca que se duplicó el uso 
de este recurso judicial en las áreas relacionadas con salud mental, neurología y psiquiatría, así como la inclusión de la atención psicológica a partir de 2017 (Tabla 4).

Tabla 4 - Tutelas de derecho a la salud y la seguridad social, 2013-2018

\begin{tabular}{l|c|c|c|c|c|c|c|c|c}
\hline Año & Total & Salud & $\%$ & $\begin{array}{c}\text { Neurol } \\
\text { ogía }\end{array}$ & \multicolumn{2}{c}{$\begin{array}{c}\text { Psiqui } \\
\text { atría }\end{array}$} & \multicolumn{3}{c}{$\begin{array}{c}\text { Psicol } \\
\text { ogía }\end{array}$} \\
\hline 2018 & 607.308 & 207.734 & 34,21 & 24.111 & 11,61 & 7.222 & 3,48 & 1.223 & 0,59 \\
2017 & 607.498 & 197.655 & 32,54 & 23.054 & 11,66 & 5.230 & 2,65 & 754 & 0,38 \\
2016 & 617.000 & 163.977 & 26,57 & 19.350 & 11,80 & 7.498 & 4,57 & ND & ND \\
2015 & 614.520 & 151.213 & 24,61 & 19.063 & 13,13 & 3.738 & 3,11 & ND & ND \\
2014 & 498.240 & 118.281 & 23,74 & 16.851 & 18,76 & 3.741 & 4,17 & ND & ND \\
2013 & 454.500 & 115.147 & 25,33 & 14.812 & 16,65 & 3.446 & 3,87 & ND & ND \\
\hline
\end{tabular}

Fuente: elaboración propia a partir de informes de Defensoría del Pueblo, 2013 2018.

Si bien se observa que en 2016 se registró el mayor incremento de solicitudes de tutela, desde que se instituyó en la Constitución Política de 1991; sin embargo, no lo fue tanto en la invocación al derecho a la salud, que fue mayor en los dos años siguientes. Asimismo, aumentaron las solicitudes de especialidades relacionadas con la salud mental (DEFENSORÍA DEL PUEBLO, 2016). Se destaca la inclusión de la psicología en los informes de 2018 y 2017; aunque en el de 2016 se declaró que la consulta psicológica estuvo entre las más solicitadas, sin indicar la cifra, junto con neurología en el régimen contributivo.

Frente a estos informes, el Ministerio de Salud (2019) emitió un boletín en el que aseveró que es impreciso generalizar porque la tutela no es el mecanismo de entrada al sistema de salud, dado que en 2018 alrededor de 25 millones de afiliados buscaron servicios de salud y el 99,7 \% de los usuarios encontró atención, sin recurrir a tutelas; mientras que estas solo representaron el 0,77 \% de las atenciones totales del país.

Asimismo, según la Encuesta Nacional de Salud Mental (2015), de la población que buscó atención entre el 88 y el 94\% lograron acceder al servicio. El porcentaje de personas que recibieron algún tipo de atención en salud mental luego de ser solicitada es de 92,7\% en la población infantil (7 a 11 años); 88,5\% en 
adolescentes (12 a 17 años); 94\% (18 a 44 años) Y 88,9\% (mayores de 44 años). El porcentaje de acceso a medicamentos es del 73,6\% (7- 12 años); 83,1\% (18-44 años) y 97,9\% (mayores de 44 años).

Según la Defensoría del Pueblo, en sus informes bianuales ha denunciado la violación sistemática de los derechos de los usuarios en cuanto a la constante negativa de acceso a las tecnologías en salud incluidas en el POS, el porcentaje de las mismas en las tutelas aún continúa alto (64,03 \%), con el agravante de que algunas de las entidades de aseguramiento que los niegan, recobran estas al Fondo de Solidaridad y Garantía del Sistema General de Seguridad en Salud FOSYGA.

Según el informe de Defensoría del Pueblo (2018), las personas que acudieron al recurso de amparo hacen parte de la población más vulnerable del país, cerca de 121.880 están en condición de desplazadas con necesidad de atención en salud. Además, el 15\% de las personas privadas de la libertad tuvieron que acudir a acción de tutela para solicitar amparo en salud, 6300 internos en 2018.

De esto da cuenta la Encuesta Nacional de Salud Mental (2015), que evidencia cómo los conflictos armados y la violencia política son considerados un problema de salud pública, que afecta de forma silenciosa la salud mental, en tanto ocasiona síndromes como el de estrés postraumático (SEPT), estallidos de ira, entumecimiento emocional, aislamiento y desesperación, desconfianza y paranoia e hipervigilancia.

El informe de Defensoría del Pueblo (2018), evidencia que lo dispuesto en la Resolución 5159 del 30 de noviembre de 2015, por la cual se adoptó el Modelo de Atención en Salud para la población privada de la libertad bajo la custodia y vigilancia del Instituto Nacional Penitenciario y Carcelario - Inpec, no ha tenido la eficacia y eficiencia esperadas, debido a que no cuentan con acceso a tecnologías en salud de manera oportuna y no tiene la efectividad en vigilancia epidemiológica para el control de enfermedades como la tuberculosis, las de transmisión sexual, de salud mental, entre otras de la salud colectiva.

Las personas con trastornos mentales presentan tasas elevadas de discapacidad y mortalidad. En Colombia, la depresión mayor unipolar (13,17\%) los 
trastornos mentales y del comportamiento debidos al uso de alcohol (8,06 \%), los trastornos de ansiedad (6,16\%) y la epilepsia (3,35\%), se encuentran entre las primeras veinte causas por años vividos con discapacidad (RODRíGUEZ; PEÑALOZA; AMAYA, 2017).

De esto da cuenta el informe de Defensoría del Pueblo de 2015, que evidencia cómo entre los usuarios más afectados se encuentran los que padecen enfermedades crónicas y de alto costo (salud mental, hipertensión arterial y cáncer, entre otras), quienes deben acudir constantemente a la tutela para que les garanticen la prestación del servicio, como también se posiciona en la Tabla 1 de principales motivos de peticiones, quejas, reclamos y denuncias PQRD de SuperSalud.

Sin embargo, aun cuando los jueces fallan a su favor, estos no son cumplidos a cabalidad por las entidades demandadas, debido a que recurren al desacato, que tampoco se sanciona como lo exige la normatividad vigente. Este hecho se evidencia en el aumento de PQRD por desacato interpuestas ante SuperSalud entre 2018 y 2019 (ver Tabla 1).

\section{Conclusiones}

La eficacia de la acción de tutela por derecho a la salud mental en la jurisprudencia colombiana, 2000-2019, se evidencia en que la tendencia ha sido la de conceder el amparo solicitado y que los demandantes hacen parte de la población con más vulnerabilidad, por tratarse de personas en situación de discapacidad; privadas de la libertad; con adicciones a SPA; en riesgo psicosocial; por considerarse un peligro para sí mismas y para sus cercanos; por la incapacidad económica y la sobrecarga de sus cuidadores, condiciones que conllevaron a que familiares y terceros acudieran en su representación para que fueran atendidos.

Aun cuando se ha establecido legislación de salud mental, incluso en atención especial como la Ley 1414 de $2010^{6}$ para el tratamiento de la epilepsia y

\footnotetext{
${ }^{6}$ Ver: REPÚBLICA DE COLOMBIA. Ley 1414 de 2010. Por la cual se establecen medidas especiales de protección para las personas que padecen epilepsia, se dictan los principios y lineamientos para su atención integral. Diario Oficial, Bogotá, n. 47.890, 11 nov. 2010. Disponible en: https://www.minsalud.gov.co/. Acceso en: 10 dic. 2019.
} 
de la implementación de la política pública, continúa el incumplimiento por parte de los prestadores del servicio de salud. Las estadísticas oficiales evidencian que, entre 2017 y 2018, cada 34 segundos se interpuso una tutela; 8 de cada 10 acciones buscaron la intervención de un juez para acceder a servicios de salud que por ley debían ser garantizados.

De la muestra de sentencias revisadas por la Corte, se destaca la reiteración de jurisprudencia por un mismo problema jurídico; en particular, el acceso a la prestación de servicios excluidos del Plan obligatorio de Salud (POS), por tratarse de población de protección especial, como las declaradas en situación de discapacidad y en adicción a sustancias psicoactivas.

De acuerdo con la Sentencia T-760-08, que acumula 22 fallos, existe una reiteración "sin que las órdenes que ha impartido la Corte, caso por caso, así como todos los jueces del país, hayan conducido a la superación de tales problemas. En realidad, los órganos responsables de lo que podría denominarse genéricamente la regulación del sistema de salud no han adoptado decisiones que les garanticen a las personas su derecho a la salud sin tener que acudir a la acción de tutela”. Como lo evidencian las fuentes analizadas, este mecanismo judicial, además de ayudar a garantizar la atención de los usuarios, es un indicador de la eficacia y eficiencia del servicio, que ha sentado precedentes en la jurisprudencia para revisar, confirmar y derogar actuaciones administrativas y técnicas que limitan o vulneran los derechos a la salud, la vida digna y la seguridad social.

Tabla 5 - Síntesis del análisis de tutelas

\begin{tabular}{|c|c|c|c|c|}
\hline Solicitudes & Derechos & Problemas & Diagnósticos & Sentencias \\
\hline $\begin{array}{l}\text { Centro } \\
\text { rehabilitación }\end{array}$ & $\begin{array}{l}\text { Derecho a la salud, } \\
\text { a la vida digna y la } \\
\text { seguridad social }\end{array}$ & $\begin{array}{l}\text { Exclusión POS. } \\
\text { Prestación del } \\
\text { servicio }\end{array}$ & $\begin{array}{l}\text { Esquizofrenia } \\
\text { paranoide. } \\
\text { Trastorno } \\
\text { afectivo bipolar. } \\
\text { Demencia por } \\
\text { consumo de } \\
\text { SPA. }\end{array}$ & $\begin{array}{l}\text { T-057/12. T-355/12. } \\
\text { T-450/16. T-497/12. } \\
\text { T-780/12. Т-578/13. } \\
\text { T-949/13. Т-124/14. } \\
\text { T-141/14. Т-153/14. } \\
\text { T-043/15. Т-632/15. } \\
\text { T-663/15. Т-010/16. }\end{array}$ \\
\hline $\begin{array}{l}\text { Centro } \\
\text { psiquiátrico }\end{array}$ & $\begin{array}{l}\text { Derecho a la salud, } \\
\text { a la vida digna y la } \\
\text { seguridad social }\end{array}$ & $\begin{array}{l}\text { Exclusión POS. } \\
\text { Prestación del } \\
\text { servicio }\end{array}$ & $\begin{array}{l}\text { Esquizofrenia } \\
\text { paranoide. } \\
\text { Epilepsia focal, } \\
\text { retardo mental } \\
\text { moderado. } \\
\text { Trastorno } \\
\text { afectivo bipolar. }\end{array}$ & $\begin{array}{l}\text { T-867/08. T-1093/08. } \\
\text { T-963/12. T-979/12. } \\
\text { T-887/13. T-949/13. } \\
\text { T-130/14. T-185/14. } \\
\text { T-714/14. T-799/14. } \\
\text { T-045/15. T-545/15. } \\
\text { T-422/17. }\end{array}$ \\
\hline
\end{tabular}




\begin{tabular}{|c|c|c|c|c|}
\hline Solicitudes & Derechos & Problemas & Diagnósticos & Sentencias \\
\hline $\begin{array}{l}\text { Suministro } \\
\text { medicamentos, } \\
\text { elementos de } \\
\text { aseo y nutrición }\end{array}$ & $\begin{array}{l}\text { Derecho a la salud, } \\
\text { a la vida digna y la } \\
\text { seguridad social }\end{array}$ & Exclusión POS & $\begin{array}{l}\text { Retraso } \\
\text { psicomotriz y } \\
\text { del lenguaje. } \\
\text { Epilepsia. } \\
\text { Retraso mental } \\
\text { moderado y } \\
\text { deterioro del } \\
\text { comportamient } \\
\text { o significativo. } \\
\text { Depresión } \\
\text { mayor }\end{array}$ & $\begin{array}{l}\text { T-409/00. T-388/03. } \\
T-1005 / 04 . \mathrm{T}-845 / 06 . \\
\mathrm{T}-760 / 08 . \mathrm{T}-209 / 13 . \\
\mathrm{T}-597 / 16 . \mathrm{T}-208 / 17\end{array}$ \\
\hline $\begin{array}{l}\text { Afiliación y } \\
\text { servicio salud } \\
\text { FFMM y Policía }\end{array}$ & $\begin{array}{l}\text { Derecho a la salud, } \\
\text { a la vida digna y la } \\
\text { seguridad social. } \\
\text { Derecho a la } \\
\text { capacidad jurídica } \\
\text { de persona en } \\
\text { condición de } \\
\text { discapacidad } \\
\text { mental. } \\
\end{array}$ & $\begin{array}{l}\text { Reconocimient } \\
\text { o de } \\
\text { discapacidad. } \\
\text { Pensión } \\
\text { invalidez. } \\
\text { Reintegro } \\
\text { servicios salud } \\
\text { FFMM y Policía }\end{array}$ & $\begin{array}{l}\text { Esquizofrenia } \\
\text { paranoide. } \\
\text { Consumo de } \\
\text { SPA. Depresión. } \\
\text { Psicosis. } \\
\text { Trastorno } \\
\text { afectivo bipolar. }\end{array}$ & $\begin{array}{l}\text { T-411/06. T-275/09. } \\
\text { T-602/09. T-377/12. } \\
\text { T-396/13. T-879/13. } \\
\text { T-195/16. T-452/18 }\end{array}$ \\
\hline $\begin{array}{l}\text { Servicio } \\
\text { especializado, } \\
\text { exámenes }\end{array}$ & $\begin{array}{l}\text { Derecho a la salud, } \\
\text { a la vida digna y la } \\
\text { seguridad social }\end{array}$ & Exclusión POS & $\begin{array}{l}\text { Retardo leve. } \\
\text { Epilepsia. } \\
\text { Trastornos del } \\
\text { comportamient } \\
\text { o, aprendizaje y } \\
\text { lenguaje. } \\
\text { Discapacidad } \\
\text { mental de } \\
\text { absoluta tipo cognitivo } \\
\end{array}$ & $\begin{array}{l}\text { T-894/13. T-482/14. } \\
\text { T-799/14. T-478/16 }\end{array}$ \\
\hline $\begin{array}{l}\text { Afiliación a EPS - } \\
\text { INPEC. Traslado } \\
\text { a Unidad de } \\
\text { Tratamiento } \\
\text { Especial. }\end{array}$ & $\begin{array}{l}\text { Derecho a la salud, } \\
\text { a la vida digna y la } \\
\text { seguridad social }\end{array}$ & $\begin{array}{l}\text { Régimen } \\
\text { excepcional } \\
\text { salud - INPEC }\end{array}$ & \begin{tabular}{|l|} 
Trastorno \\
afectivo bipolar. \\
Consumo de \\
SPA. \\
Discapacidad \\
mental \\
absoluta. \\
Esquizofrenia \\
paranoide. \\
Ansiedad. \\
Trastorno \\
depresivo \\
mayor.
\end{tabular} & $\begin{array}{l}\text { T-744/09. T-377/12. } \\
\text { T-750A/12. T-149/14. } \\
\text { T-016/17. }\end{array}$ \\
\hline $\begin{array}{l}\text { Atención } \\
\text { domiciliaria }\end{array}$ & $\begin{array}{l}\text { Derecho a la salud, } \\
\text { a la vida digna y la } \\
\text { seguridad social }\end{array}$ & Exclusión POS & \begin{tabular}{|l} 
Trastorno \\
mental. \\
Esquizofrenia \\
paranoide
\end{tabular} & T-886/12. T-454/14 \\
\hline $\begin{array}{l}\text { Transporte } \\
\text { especial }\end{array}$ & $\begin{array}{l}\text { Derecho a la salud, } \\
\text { a la vida digna y la } \\
\text { seguridad social }\end{array}$ & Exclusión POS & $\begin{array}{l}\text { Cuadriplejia. } \\
\text { Autismo. } \\
\text { Epilepsia } \\
\text { parálisis } \\
\text { cerebral. } \\
\text { Hipotonía y } \\
\text { retardo global } \\
\text { del desarrollo. }\end{array}$ & $\begin{array}{l}\text { T-155/14. T-076/15. } \\
\text { T-074/17 }\end{array}$ \\
\hline
\end{tabular}




\begin{tabular}{|c|c|c|c|c|}
\hline Solicitudes & Derechos & Problemas & Diagnósticos & Sentencias \\
\hline $\begin{array}{l}\text { Interrupción } \\
\text { voluntaria } \\
\text { embarazo }\end{array}$ & $\begin{array}{l}\text { Derechos } \\
\text { reproductivos }\end{array}$ & $\begin{array}{l}\text { Orden } \\
\text { procedimiento } \\
\text { IVE }\end{array}$ & $\begin{array}{l}\text { Trastorno mixto } \\
\text { de ansiedad. } \\
\text { Depresión. } \\
\text { Angustia. }\end{array}$ & $\begin{array}{l}\text { T-585/10. T-841/11. } \\
\text { T-532/14. T-731/16. }\end{array}$ \\
\hline $\begin{array}{l}\text { Traslado lugar } \\
\text { de empleo }\end{array}$ & $\begin{array}{l}\text { Derecho a la salud, } \\
\text { a la vida digna y la } \\
\text { seguridad social }\end{array}$ & $\begin{array}{l}\text { Salud mental } \\
\text { en el trabajo }\end{array}$ & \begin{tabular}{|l|} 
Trastorno \\
depresivo. \\
Trastorno \\
afectivo bipolar. \\
Trastorno de \\
ansiedad por \\
estrés laboral. \\
\end{tabular} & $\begin{array}{l}\text { T-882/06. T-372/12. } \\
\text { T-095/13. T-042/14. }\end{array}$ \\
\hline $\begin{array}{l}\text { Restablecimient } \\
\text { o de derechos } \\
\text { por interdicción }\end{array}$ & $\begin{array}{llr}\text { Derecho a la } & \text { la } \\
\text { capacidad juridica } \\
\text { de persona } & \text { en } \\
\text { condición } & \text { de } \\
\text { discapacidad } & \\
\text { mental. } & \\
\end{array}$ & Interdicción & $\begin{array}{l}\text { Discapacidad } \\
\text { mental } \\
\text { absoluta. } \\
\text { Contenido } \\
\text { delirante }\end{array}$ & $\begin{array}{l}\text { T-984/01. T-1019/04. } \\
\text { T-684/14. }\end{array}$ \\
\hline Hogar geriátrico & $\begin{array}{l}\text { Derecho a la salud, } \\
\text { a la vida digna y la } \\
\text { seguridad social }\end{array}$ & Exclusión POS & $\begin{array}{l}\text { Demencia senil. } \\
\text { Trastorno } \\
\text { afectivo bipolar }\end{array}$ & T-949/13. T-024/14 \\
\hline $\begin{array}{l}\text { Solicitud } \\
\text { cuidador }\end{array}$ & $\begin{array}{l}\text { Derecho a la salud, } \\
\text { a la vida digna y la } \\
\text { seguridad social }\end{array}$ & Exclusión POS & $\begin{array}{|ll|}\text { Epilepsia } & \text { y } \\
\text { parálisis } & \\
\text { cerebral. } & \\
\text { Alzhéimer. } & \\
\end{array}$ & T-096/16. T-120/17 \\
\hline $\begin{array}{l}\text { Procedimiento } \\
\text { anticoncepción } \\
\text { quirúrgico }\end{array}$ & 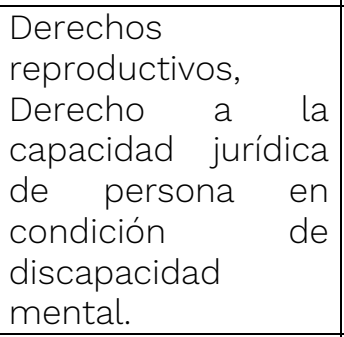 & $\begin{array}{l}\text { Autorización } \\
\text { judicial }\end{array}$ & $\begin{array}{l}\text { Retraso mental } \\
\text { leve y epilepsia. }\end{array}$ & T-063/12. T-303/16 \\
\hline $\begin{array}{l}\text { Diagnóstico } \\
\text { médico y } \\
\text { atención integral } \\
\text { víctimar del } \\
\text { conflicto }\end{array}$ & $\begin{array}{l}\text { Derecho a la salud, } \\
\text { a la vida digna y la } \\
\text { seguridad social }\end{array}$ & $\begin{array}{l}\text { Reconocimient } \\
\text { o de situación } \\
\text { psicosocial }\end{array}$ & \begin{tabular}{|l|} 
Depresión \\
profunda. \\
Angustia. \\
Retardo mental \\
severo. \\
\end{tabular} & T-045/10. T-050/19 \\
\hline $\begin{array}{lr}\text { Reintegro } & \text { de } \\
\text { dinero } & \text { por } \\
\text { urgencia } & \\
\text { psiquiátrica } & \\
\end{array}$ & $\begin{array}{l}\text { Derecho a la salud, } \\
\text { a la vida digna y la } \\
\text { seguridad social }\end{array}$ & Exclusión POS & \begin{tabular}{|lr|}
\multicolumn{2}{|l|}{ Depresión. } \\
Retardo & leve. \\
Intento & de \\
suicidio. & \\
\end{tabular} & $\mathrm{T}-134 / 02$ \\
\hline $\begin{array}{l}\text { Condonación de } \\
\text { deuda por } \\
\text { discapacidad } \\
\text { mental }\end{array}$ & $\begin{array}{llr}\text { Derecho a la } \\
\text { capacidad jurídica } \\
\text { de persona en } \\
\text { condición } & \text { de } \\
\text { discapacidad } & \\
\text { mental. } & \\
\end{array}$ & Interdicción & $\begin{array}{l}\text { Discapacidad } \\
\text { mental } \\
\text { absoluta }\end{array}$ & T-933/13 \\
\hline
\end{tabular}

Fuente: elaboración propia, a partir de sentencias de tutela de la Corte Constitucional de Colombia

\section{Agradecimientos}

Este artículo se deriva del proyecto Estudios Sociales y Culturales en Salud Mental, financiado por convocatoria de Vicerrectoría de Investigaciones de la Universidad Católica Luis Amigó, 2019. Código: 55860. 


\section{Referencias}

AITKEN, María. Prólogo. In: Hernández, Nelly.; Méndez, Gilma.; Soto, Carolina. La salud mental: una cuestión de derechos humanos. Modelo pedagógico para intervención con grupos de población vulnerable. Ibagué: Universidad del Tolima, 2011.

ARDILA, Sara. La salud mental a la luz de la Constitución colombiana: análisis de algunas sentencias de la Corte Constitucional, 1992-2009. Rev. Colomb. Psiquiat., Bogotá, v. 39, n. 3, p. 493-509, 2010. Disponible en: https://doi.org/10.1016/S0034-7450(14)60221-3. Acceso en: 10 dic. 2019.

COMISIÓN DE REGULACIÓN DE SALUD. Acuerdo 029 de 2011. Metodología para la actualización integral del Plan obligatorio de salud del Sistema general de Seguridad social en Salud. Bogotá: Minsalud, 2011. Disponible en: https://www.minsalud.gov.co

COMISIÓN DE REGULACIÓN DE SALUD. Acuerdo 028 de 2011. Por el cual se define, aclara y actualiza integralmente el Plan Obligatorio de Salud. Bogotá: Minsalud, 2011. Disponible en: https://www.minsalud.gov.co. Acceso en: 10 dic. 2019.

CONGRESO DE LA REPÚBLICA. Ley 100 de 1993. Por la cual se crea el sistema de seguridad social integral y se dictan otras disposiciones. Diario Oficial, Bogotá, n. 41.148, 23 dic. 1993. Disponible en:

http://www.secretariasenado.gov.co/senado/basedoc/ley_0100_1993.html. Acceso en: 10 dic. 2019.

CONGRESO DE LA REPÚBLICA. Ley 1616 de 2013 Por medio de la cual se expide la Ley de Salud Mental... Diario Oficial, Bogotá, n. 48.680, 21 enero 2013. Disponible en:

https://www.minsalud.gov.co/sites/rid/Lists/BibliotecaDigital/RIDE/DE/DIJ/ley1616-del-21-de-enero-2013.pdf. Acceso en: 10 dic. 2019.

CONGRESO DE LA REPÚBLICA. Ley Estatutaria 1751 de 2015. Por medio de la cual se regula el derecho fundamental a la salud y se dictan otras disposiciones. Diario Oficial, Bogotá, n. 49.427, 16 feb. 2015. Disponible en: https://www.minsalud.gov.co. Acceso en: 10 dic. 2019. 
CORCHO, Carolina. Poderes fácticos y cooptación en el sistema de salud en Colombia: El caso de Medimás. Fundación Heinrich Böll, Bogotá, n. 12, 2018. Série Ideas Verdes. Disponible en:

https://co.boell.org/es/2018/11/09/poderes-facticos-y-cooptacion-en-el-

sistema-de-salud-en-colombia-el-caso-de-medimas. Acceso en: 10 dic. 2019.

DEFENSORÍA DEL PUEBLO. La tutela y los derechos a la salud y a la seguridad social. Bogotá: Defensoría del Pueblo, 2018. Disponible en:

http://www.defensoria.gov.co/public/pdf/Tutela-los-derechos-de-la-salud2018.pdf. Acceso en: 10 dic. 2019.

DEFENSORÍA DEL PUEBLO. La tutela y los derechos a la salud y a la seguridad social. Bogotá: Defensoría del Pueblo, 2017. Disponible en:

http://desarrollos.defensoria.gov.co/desarrollo1/ABCD/bases/marc/documentos/ textos/La_Tutela_y_el_Derecho_a_la_salud_2017.pdf. Acceso en: 10 dic. 2019.

DEFENSORÍA DEL PUEBLO. La tutela y los derechos a la salud y a la seguridad social. Bogotá: Defensoría del Pueblo, 2016. Disponible en:

http://desarrollos.defensoria.gov.co/desarrollo1/ABCD/bases/marc/documentos/ textos/La_tutela_y_el_derecho_a_la_salud_2016.PDF. Acceso en: 10 dic. 2019.

\section{DEFENSORÍA DEL PUEBLO. La tutela y los derechos a la salud y a la seguridad} social. Bogotá: Defensoría del Pueblo, 2015. Disponible en:

http://desarrollos.defensoria.gov.co/desarrollo1/ABCD/bases/marc/documentos/ textos/La_Tutela_y_los_Derechos_a_la_Salud_y_a_la_Seguridad_Social_2015_co mpleto_(1).pdf . Acceso en: 10 dic. 2019.

DEFENSORÍA DEL PUEBLO. La tutela y los derechos a la salud y a la seguridad social. Bogotá: Defensoría del Pueblo, 2014. Disponible en:

http://desarrollos.defensoria.gov.co/desarrollo1/ABCD/bases/marc/documentos/ textos/La\%20tutela\%20y\%20los\%20derechos\%20a\%20la\%20salud\%20y\%20a\%2 Ola\%20seguridad\%20social\%202014.pdf. Acceso en: 10 dic. 2019.

DEFENSORÍA DEL PUEBLO. La tutela y los derechos a la salud y a la seguridad social. Bogotá: Defensoría del Pueblo, 2013. Disponible en:

http://desarrollos.defensoria.gov.co/desarrollo1/ABCD/bases/marc/documentos/ textos/La\%20tutela\%20y\%20los\%20derechos\%20a\%20la\%20salud\%20y\%20a\%2 Ola\%20seguridad\%20social\%202013.pdf. Acceso en: 10 dic. 2019. 
ECHEVERRI, María. Indignación justa: estudios sobre la acción de tutela en salud en Medellín. Medellín: Facultad Nacional de Salud Pública: Hombre nuevo editores, 2013.

MINISTERIO DE SALUD. Acuerdo 008 de 1994. Por el cual se adopta el Plan Obligatorio de Salud para el Régimen Contributivo del Sistema General de Seguridad Social en Salud. Bogotá: Minsalud, 1994. Disponible en: https://www.minsalud.gov.co. Acceso en: 10 dic. 2019.

MINISTERIO DE SALUD. Resolución 2358 de 1998. Por la cual se adopta la Política Nacional de Salud. Diario Oficial, Bogotá, n. 43.338, 13 jul. 1998. Disponible en: https://www.minsalud.gov.co. Acceso en: 10 dic. 2019.

MINISTERIO DE SALUD Y PROTECCIÓN SOCIAL. Resolución 4886 de 2018. Por la cual se adopta la Política Nacional de Salud Mental. Diario Oficial, Bogotá, n. 50770, 7 noviembre 2018. Disponible en:

https://www.minsalud.gov.co/Normatividad_Nuevo/Resoluci\%C3\%B3n\%20No.\%2 04886\%20de\%202018.pdf. Acceso en: 10 dic. 2019.

MINISTERIO DE SALUD Y PROTECCIÓN SOCIAL. Resolución 5857 de 2018. Por la cual se actualiza integralmente el plan de beneficios en salud con cargo a la Unidad de Pago por Capitación, UPC. Diario oficial, Bogotá, n. 50818, 26 enero 2019. Disponible en: https://www.minsalud.gov.co. Acceso en: 10 dic. 2019.

MINISTERIO DE SALUD Y PROTECCIÓN SOCIAL. Resolución 5592 de 2015. Por la cual se actualiza integralmente el Plan de Beneficios en Salud con cargo a la Unidad de Pago por Capitación (UPC) del Sistema General de Seguridad Social en Salud (SGSSS) y se dictan otras disposiciones. Diario Oficial, Bogotá, n. 49739, 28 diciembre 2015. Disponible en: https://www.minsalud.gov.co Acceso en: 10 dic. 2019.

MINISTERIO DE SALUD. Encuesta Nacional de Salud Mental. Bogotá: Minsalud, 2015. Disponible en: https://www.minsalud.gov.co/. Acceso en: 10 dic. 2019.

MINISTERIO DE SALUD. La tutela no es el mecanismo de entrada al sistema de salud. Boletín de Prensa, Bogotá, n. 110, 2019. Disponible en:

https://www.minsalud.gov.co/Paginas/La-tutela-no-es-el-mecanismo-deentrada-al-sistema-de-salud-Minsalud.aspx. Acceso en: 10 dic. 2019. 
RODRÍGUEZ, Jesús.; PEÑALOZA, Rolando.; AMAYA, Jeannette. Estimación de la carga global de enfermedad en Colombia 2012: nuevos aspectos metodológicos. Rev. Salud Pública, Bogotá, v. 19, n. 2, p. 235-240, 2017. Disponible en: http://dx.doi.org/10.15446/rsap.v19n2.66179. Acceso en: 10 dic. 2019.

REPÚBLICA DE COLOMBIA. Constitución Política. Gaceta Constitucional, Bogotá, n. 116, 20 jul. 1991a. Disponible en:

http://www.secretariasenado.gov.co/senado/basedoc/constitucion_politica_1991. html. Acceso en: 10 dic. 2019.

REPÚBLICA DE COLOMBIA. Decreto 2591. Por el cual se reglamenta la acción de tutela consagrada en el artículo 86 de la Constitución Política. Bogotá:

Presidencia da República, 1991b. Disponible en:

https://www.funcionpublica.gov.co/eva/gestornormativo/norma.php?i=5304.

Acceso en: 10 dic. 2019.

REPÚBLICA DE COLOMBIA. Ley 1414 de 2010. Por la cual se establecen medidas especiales de protección para las personas que padecen epilepsia, se dictan los principios y lineamientos para su atención integral. Diario Oficial, Bogotá, $n$. 47.890, 11 nov. 2010. Disponible en: https://www.minsalud.gov.co/. Acceso en: 10 dic. 2019. 